\title{
Multilayer Interlocked Woven Fabrics: Simulation of RTM Mold Filling Operation with Preform Permeability Properties
}

\author{
Naveen V Padaki ${ }^{1}$, R. Alagirusamy ${ }^{2 *}$, B.L. Deopura ${ }^{2}$ and R. Fangueiro ${ }^{3}$ \\ ${ }^{1}$ Regional Silk Technological Research Station, CSTRI, Central Silk Board, \\ Muga farm, Khanapara, Guwahati-781022, India \\ ${ }^{2}$ Department of Textile Technology, Indian Institute of Technology-Delhi, \\ Hauz khas, New Delhi - 110 016, India \\ ${ }^{3}$ Department of Textile Engineering, University of Minho, \\ Guimarães-4800 058, Portugal
}

\begin{abstract}
The simulation of resin flow during the resin transfer molding (RTM) process through multilayered textile fabric of known permeability and porosity has been attempted in this study. A simple three-dimensional computational fluid dynamics (CFD) simulation model has been developed and the results of the simulation are compared with the experimental RTM resin flow through multilayer interlocked woven structures. A multiphase simulation model is observed to reasonably predict the time for RTM mold filling. Fabric structural influence in terms of an Interlacement Index (I) has significant influence on the resin flow behaviour of the multilayered preform. A higher $I$ of the preform means a longer time to fill the mold in both the experimental and simulated results. Images of the simulated flow front has been compared with the experimental results and it is observed that not only the mold filling time, but also the area of resin flow in the multilayer perform, is influenced by a fabric structural factor, $I$.
\end{abstract}

Keywords: Multilayer Interlocked Structures, Interlacement Index, Permeability, RTM Simulation

\section{Introduction}

Preform engineering plays an important role in the design of composites through optimization of the design elements, which has received widespread attention among researchers in recent times (Weimer, 2003).

Woven preforms are the key reinforcements used for composites and most of the composites are made by stacking layers of woven preforms one over the other which lead to prominent delamination failure in the composite materials. Sewing and three dimensional (3D) weaving are promising technologies which address the short comings of layer reinforced composites (Naveen et al., 2008).

Multilayer interlocked fabrics are a quite distinct

\footnotetext{
* Corresponding author. Tel.: 0091-11-26591419; Fax: 0091-11-26581103 E-mail address: alagiru@gmail.com (R. Alagirusamy)
}

class of 3D preforms, which have been scantily explored to achieve interlocking of fabric layers during the weaving stage. They provide the advantage of cost effective preform manufacture with control over layer interlock density apart from imparting higher impact and delamination resistance to fibre reinforced composites ( $\mathrm{Hu}$, 2000; Hu et al., 2002).

Multilayer interlocked fabrics are composed of several series of warp and weft yarns that form distinct layers which are bound by interlacing warp ends (Adanur et al., 1997).

Based on the type of interlacements, the multilayer fabrics are catagorised into angle interlocked and layer interlocked structures. In angle-interlock structures, warp yarns of each layer interlace with the weft yarns of the adjacent layers, while in layer-interlock structures, warp yarns interlace the top and bottom layers of the fabric. 


\section{Interlacement Index (I) as a Factor that Represents Textile Structure}

Woven preforms have two sets of yarns perpendicular to each other, interlaced by a weaving process. It has been suggested that woven fabric areas should be divided into three types of fields (Milasius, 2000), which are contact (c), interlacing (i) and float (f). A c field is defined as the projected region occupied by warp and weft thread systems. An i field is a region where there is a cross-over of warp yarn from one plane to another due to weaving around a weft yarn, and vice versa. When the yarn does not shift from one plane to another between two c fields, then it can be termed as an $\mathrm{f}$ field.

A generalization of the woven structure by an integrated factor based on interlacements for structure-property correlation has been proposed (Naveen et al., 2009) as the Interlacement Index (I). $I$ is defined as a ratio of the number of interlacements in a given weave repeat to the maximum possible $\mathrm{c}$ fields in the design as given by Equation (1), where $i_{\mathrm{wp}}$ and $\mathrm{i}_{\mathrm{wf}}$ are interlacements in warp and weft, respectively. A warp and weft repeat $\left(\begin{array}{lll}R_{1} \times & R_{2}\end{array}\right)$ product with a woven design gives the maximum possible $\mathrm{c}$ fields in a woven design repeat. A plain woven structure has the highest interlacement $(I=2)$ and a non-interlaced structure would have an $I$ value of 0 .

$$
I=\left(\frac{i_{w p}+i_{w f}}{R_{1} \cdot R_{2}}\right)
$$

Variations in the interlacements of a multilayer interlocked structure alter not only the fabric geometry, but also preform properties, such as permeability, porosity and compressibility, which in turn, influence the resin flow characteristics through the preform for composite manufacture processes, such as resin transfer molding (RTM). RTM is a closed mold process for making composite materials to produce parts more cost effectively than other composite manufacturing methods. However, the resin flow in RTM through a reinforcement tends to be unpredictable for complex shaped composite material. Hence, computational fluid dynamics (CFD) simulation techniques are adopted as a predictive method for resin flow to design RTM molds and determine the vent locations in the molds. Resin parameters and preform characteristics influence the composite material manufacture by RTM processes. Accurate prediction of the resin flow through the preform inside the RTM mold is one of the most critical aspects of an RTM simulation program. Permeability, compressibility and formability are prominent preform properties among which the first two are crucial input parameters for the simulation of resin impregnation by an RTM process (Verleye, 2008).

There are two common approaches for the development of a simulation model for resin flow through the preforms for an RTM process. The first approach is to combine a porous media theory with more generalized flow models, such as the Stokes flow, to take into account micro-geometric effects. This approach is referred to as micro-modeling. Another approach is to use a porous media theory and apply it to the entire mold which results in a macro-flow model. Micro-flow models can take into account complex geometry regions of a mold, while macro-flow models can consider larger, more complex molds (Crains et al., 1999).

The micro model separates flow into two regions, channels and fiber tows. Channel permeability in all three directions are calculated from general flow equations such as Stokes flow, whereas permeability along the length of fiber tows are calculated experimentally and permeability transverse to the fiber bundles are calculated by using the Kozeny-Carmen equation given by Equation (2).

$u=\frac{R^{2} \varepsilon^{7}}{c(-s)^{2}} \cdot \frac{\Delta P}{\mu}$

Where

u-volume average velocity,

$\mathrm{R}$ - fibre radius,

c- shape factor,

$\varepsilon$ - fabric porosity,

$\Delta \mathrm{P}$-pressure difference, and

$\mu$ - fluid viscosity

Different cells that correspond to either fibers or channels are then stacked according to the preform 
geometry and resin flow through the cells is solved by using Stokes flow or methods such as the Gauss-Seidel difference scheme (Crains et al., 1999).

Macro-flow models consider Darcy's law for resin flow through a fibrous preform given by Equation (3), by grouping all layers of reinforcement and giving them a single permeability value.

$Q=-\frac{K \Delta P}{L}$

where $\mathrm{Q}$ is the flow rate, $\mathrm{K}$ is a flow conductivity proportionality constant or permeability constant of the porous medium with respect to the fluid, $\Delta \mathrm{P}$ is the net flow pressure head and $\mathrm{L}$ is the length of the capillary flow (Patnaik et al., 2006).

One such macro-modeling program is the Liquid Injection Molding Software (LIMS) developed by the University of Delaware's Center for Composite Materials (Kruckenberg et al., 1998).

Another general type of CFD software is the Fluent software which solves the integral form of the time-dependent Reynolds-averaged Navier-Stokes equations in three dimensions using a structured grid design. For all flows, the Fluent solves conservation equations for mass and momentum. The objectives of the present work are to simulate the RTM resin flow using determined preform permeability properties as input through the Fluent software, compare the flow predictions with experimental data and analyze the RTM flow behaviour with respect to a multilayer structure.

\section{Flow Model Considerations}

The flow model has two main input variables, namely, pressure at the outlet and fabric porosity. The fluid pressure accuracy is determined by the mesh resolution in the direction of the pressure gradients. The pressure gradients will typically be the highest around sharp geometry transitions, such as inlet and outlet locations in the case of the RTM. The resolution of the flow front location will be determined by the distance from one node to the next. Since the multiphase model (MM) with the volume of fluid (VOF) method locates the flow front, the uncertainty in the flow front location is the length of the element. The assumptions made in the formulation of the model include:

1. The preform is a homogeneous, porous and isotropic medium.

2. The flow is in a quasi-steady state.

3. Capillary and inertial effects are neglected (low Reynolds number flow).

4. The fluid is assumed to be Newtonian (its viscosity is independent of shear rate), and incompressible.

5. The fluid does not leak from the mold cavity.

The governing differential equation (4) can be expressed by Darcy's law in the following form (Verleye, 2008):

$\frac{\partial}{\partial x_{i}}\left(\frac{k}{\mu} \frac{\partial P}{\partial x_{j}}\right)=0$

where $\mu$ is the fluid viscosity, $k$ is the permeability tensor of the preform, and $\mathrm{P}$ is the fluid pressure.

\subsection{Model Description}

A 3D multiphase CFD model was built to simulate the resin flow through multilayer preforms during an RTM operation. Prior to resin injection inside the mold through the preform, there is air, or void space if it is drawn through a vacuum. During injection, there are multiple flow areas with resin, and fibers in one region and fibers and air or void spaces in another. Many times, a transition region exists between the two where air and resin coexist in varying concentrations. Hence, a multiphase flow model has been developed for the RTM flow simulation. This model not only provides visuals of resin flow (flow front) in the mold, but also depicts the transient behaviour of the flow of two phases (air and resin) where the initial air phase is replaced by the resin during the mold filling process. The interphase of such a multiphase is modeled by using VOF, which is a multiphase option in the Fluent software.

The tracking of the flow front or the interface between the phases is accomplished by the solution of a continuity equation for the volume 
fraction of one (or more) of the phases in the VOF formulation. For the ' $q$ 'th phase, Equation (5) is given by:

$$
\frac{3 a_{q}}{\partial t}+\vec{v} \cdot \nabla c_{q}=\frac{s_{q_{q}}}{f_{q}}
$$

where, $\alpha_{\mathrm{q}}$ is the volume fraction of the ' $\mathrm{q}$ 'th phase and $S_{\alpha q}$ is the mass of the ' $q$ 'th phase (Fluent Tutorial Guide, 2003).

\subsection{Model Geometry}

A model geometry that comprise fabric that is 200 $\mathrm{mm} \times 200 \mathrm{~mm}$ in size with an inlet and outlet that are both $4.5 \mathrm{~mm}$ on opposite sides of the fabric and $10 \mathrm{~mm}$ away from the fabric edge, was created by using GAMBIT software (V 2.0.4). The illustration of the model geometry of the fabric is given below (Figure 1). Two geometries were created with a fabric thickness of $1.0 \mathrm{~mm}$ and $1.5 \mathrm{~mm}$.

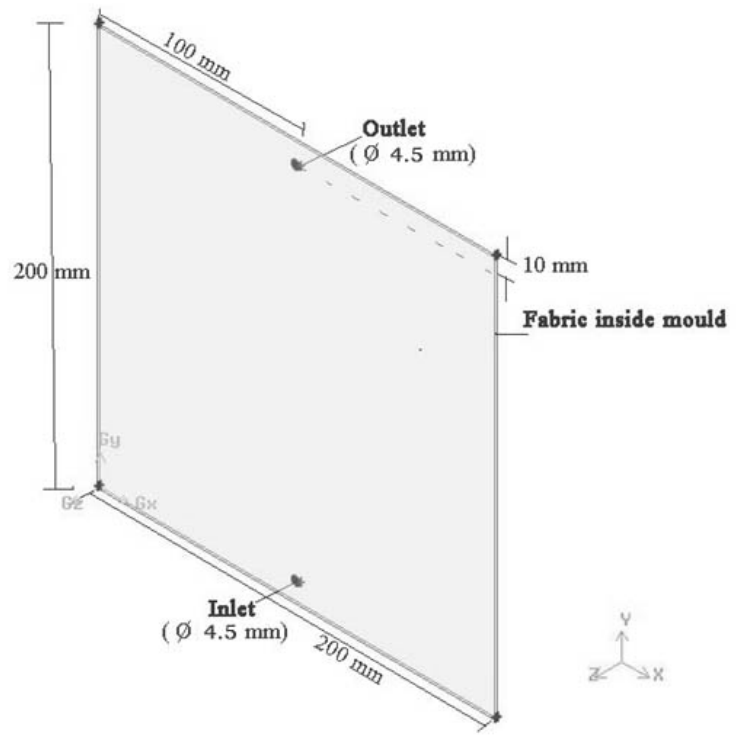

Fig. 1. Model Geometry of the Fabric

\subsection{Mesh Generation}

The Cooper scheme was used to automatically generate an unstructured, hexahedral mesh. The advantage of unstructured grid methods is that they are automated and therefore, require little user time or effort. Unstructured methods also enable the solution of large and detailed problems in minutes or hours instead of days or weeks in contrast to structured grid generation methods. A hexahedron provides shape functions with additional terms that may increase the accuracy of the solution. It also provides directional sizing without losing accuracy. For example, a very thin hexahedron within a boundary layer for fluid flow calculations performs far better than a thin tetrahedron. A hexahedral mesh decreases the overall element count. A tetrahedral mesh usually increases the element count 4 - to 10 -fold over a hexahedral mesh.

A fabric volume mesh was created for a thickness of $1 \mathrm{~mm}$, with 80,646 nodes and 39,922 elements where both inlet and outlet face meshes consisted of 33 elements and 25 nodes each. Similarly, a fabric volume mesh for a thickness of $1.5 \mathrm{~mm}$ comprised 120,969 nodes and 79,844 elements where both inlet and outlet face meshes consisted of 33 elements and 25 nodes each. The meshed structure of the fabric model geometry with a thickness of $1 \mathrm{~mm}$ is illustrated by Figure 2. The mesh was examined by using a GAMBIT inbuilt tool to assess the quality of the resultant mesh, as properties such as skewness can greatly affect the accuracy and robustness of the CFD solution. The range of the skewness of the 3D element (brick) was examined for equiangle skew and the results are tabulated in Table 1. In quality assessments of the mesh for skewness, smaller range values are more desirable, which are observed in the meshed geometry with more than $95 \%$ elements in the range of $0-0.2$. The file was saved into the Fluent 6 solver with fabric volume as fluid type material.

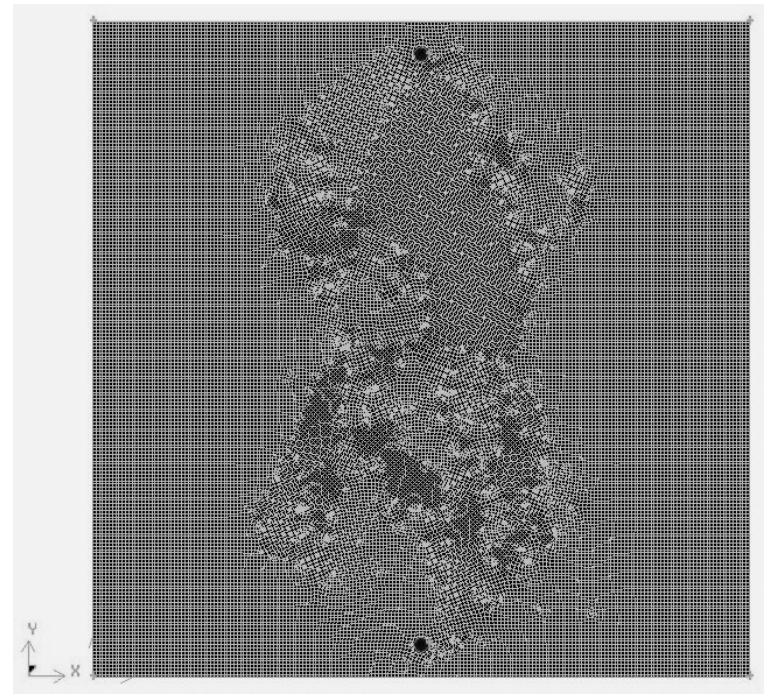

Fig. 2. Meshed Fabric Geometry with Thickness of $1 \mathrm{~mm}$ 
Table 1. Mesh Examination Values

\begin{tabular}{ccc}
\hline \multirow{2}{*}{$\begin{array}{c}\text { Skewness Range } \\
\text { (Equiangle skew) }\end{array}$} & \multicolumn{2}{c}{ Active elements (\%) } \\
\cline { 2 - 3 } & $1 \mathrm{~mm}$ & $1.5 \mathrm{~mm}$ \\
\hline $0-0.1$ & 88.34 & 89.52 \\
$0.1-0.2$ & 7.12 & 7.14 \\
$0.2-03$ & 2.21 & 1.92 \\
$0.3-0.4$ & 1.83 & 1.06 \\
$0.4-0.5$ & 0.47 & 0.36 \\
\hline
\end{tabular}

\subsection{Multiphase Model}

A multiphase flow model based on the above grid was developed for the RTM flow simulation.

\subsection{Grid Import and Model Selection}

The mesh geometry file was imported into the Fluent 3D solver; the imported grid was checked and scaled to actual units of measurements. A segregated solver (default) was selected for the incompressible resin flow through fabric during the RTM process (low velocities of the fluid-low Reynolds number). The $1^{\text {st }}$ order implicit, physical velocity porous formulation for 3D unsteady flow was opted in the model-solver options. A viscous laminar model was selected for the physical model (laminar flow).

\subsection{Material Properties and Boundary Conditions}

The two-layer (2-ply) and three-layer (3-ply) interlocked multilayer fabric samples were woven on a 4 harness, flexible rapier automatic loom (Dornier), at a setting of $400 \mathrm{rpm}$ with $24 \mathrm{ends} / \mathrm{cm}$ and 12 picks $/ \mathrm{cm}$. Five meter lengths of 4 varieties of nylon 2-ply fabrics (N2P1-4) and 5 varieties of 3-ply fabrics (N3P1-5) were woven for the present study by using high tenacity nylon-6 filament yarn (96 Tex, fibre diameter $27.2 \mu \mathrm{m}$ ). The graphical representation of the woven design and 2-ply and 3-ply multilayer woven structures are represented in Figures 3 and 4.

The general construction characteristics of the fabric samples made are provided in Table 2, where $s_{1}, s_{2}$ denote warp $/ \mathrm{cm}$ and weft $/ \mathrm{cm}$, respectively, and $\mathrm{c}_{1}, \mathrm{c}_{2}$ represent warp and weft crimp \% values, respectively. Apart from regular interlacing warp yarns, multi-layer structures $\mathrm{N} 2 \mathrm{P} 1, \quad \mathrm{~N} 2 \mathrm{P} 2, \mathrm{~N} 2 \mathrm{P} 3, \mathrm{~N} 3 \mathrm{P} 1, \mathrm{~N} 3 \mathrm{P} 2$ and N3P3 contain a relatively straighter set of warp yarns in the structure, due to which, the crimp values of warp yarns in these structures have higher variations.

Table 2. Multilayer Woven Interlocked Perform Properties

\begin{tabular}{cccccc}
\hline $\begin{array}{c}\text { Sample } \\
\text { Code }\end{array}$ & $\begin{array}{c}\mathrm{s}_{1} \times \mathrm{s}_{2}, \\
/ \mathrm{cm}\end{array}$ & $\begin{array}{c}\mathrm{c}_{1}, \\
\%\end{array}$ & $\begin{array}{c}\mathrm{c}_{2}, \\
\%\end{array}$ & $\begin{array}{c}\text { Thickness } \\
\mathrm{mm}\end{array}$ & $\begin{array}{c}\text { Aerial Density, } \\
\mathrm{g} / \mathrm{m}^{2}\end{array}$ \\
\hline $\mathrm{N} 2 \mathrm{P} 1$ & $24 \times 13$ & 7.8 & 3.6 & 1.12 & $432.6(2.8)$ \\
$\mathrm{N} 2 \mathrm{P} 2$ & $23 \times 11$ & 6.9 & 3.2 & 1.18 & $411.4(3.1)$ \\
$\mathrm{N} 2 \mathrm{P} 3$ & $23 \times 11$ & 5.3 & 2.1 & 1.2 & $407.6(5.3)$ \\
$\mathrm{N} 2 \mathrm{P} 4$ & $24 \times 12$ & 4.3 & 2.6 & 1.25 & $412.2(4.7)$ \\
$\mathrm{N} 3 \mathrm{P} 1$ & $25 \times 12$ & 6.3 & 3.1 & 1.22 & $422.2(2.9)$ \\
$\mathrm{N} 3 \mathrm{P} 2$ & $24 \times 12$ & 4.4 & 2.1 & 1.34 & $420.1(3.2)$ \\
$\mathrm{N} 3 \mathrm{P} 3$ & $24 \times 12$ & 5.6 & 3.0 & 1.32 & $438.5(2.5)$ \\
$\mathrm{N} 3 \mathrm{P} 4$ & $24 \times 13$ & 6.5 & 4.0 & 1.27 & $436.8(1.4)$ \\
$\mathrm{N} 3 \mathrm{P} 5$ & $25 \times 12$ & 6.8 & 3.6 & 1.41 & $437.2(2.9)$ \\
\hline
\end{tabular}




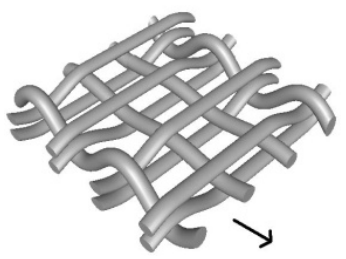

$\mathrm{N} 2 \mathrm{P} 1$

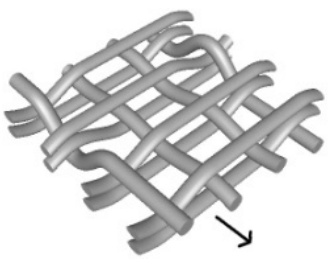

$\mathrm{N} 2 \mathrm{P} 3$

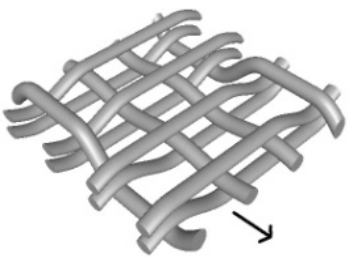

$\mathrm{N} 2 \mathrm{P} 2$

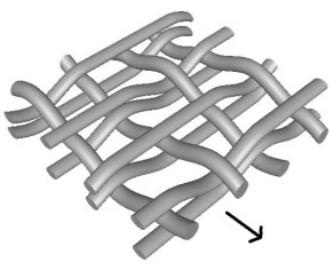

$\mathrm{N} 2 \mathrm{P} 4$
Fig. 3. Two-layer interlocked woven structures $(\rightarrow$ warp direction)

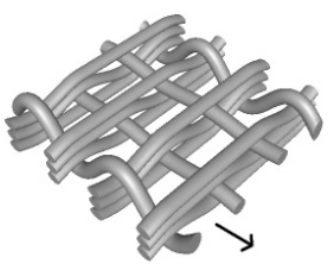

N3P1

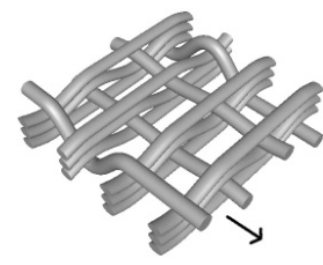

N3P2

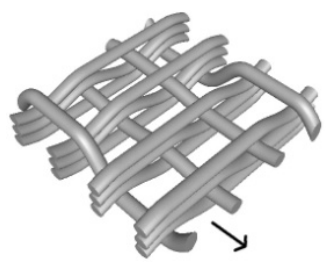

N3P3

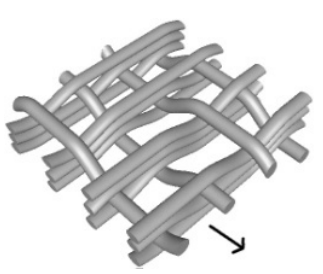

N3P4

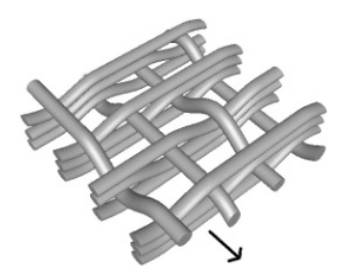

N3P5
Fig. 4. Three-layer interlocked woven structures $(\rightarrow$ warp direction)

Resin was created with a specified density (1140 $\left.\mathrm{kg} / \mathrm{m}^{3}\right)$ and viscosity $(0.60 \mathrm{~kg} / \mathrm{m}-\mathrm{s})$ along with the existing 'air' (density $1.225 \mathrm{~kg} / \mathrm{cc}$, viscosity $(1.7894 \mathrm{e}-5 \mathrm{~kg} / \mathrm{m}-\mathrm{s})$. The multiphase VOF option was selected under the model option; air was defined as the primary phase; and resin was set as secondary phase. Gravity $\left(-9.81 \mathrm{~m} / \mathrm{s}^{2}\right)$ was activated in the operating conditions panel in the
' $\mathrm{z}$ ' direction; the density of air $(1.225 \mathrm{~kg} / \mathrm{cc})$ was specified under the variable density parameter for better convergence of solution. Mixed mode (both for air and resin) boundary conditions for the inlet (pressure inlet, 0 pascal) and outlet (pressure outlet, -97325 pascals in ' $z$ ' direction) were set. Mixed mode fabric permeability (viscous resistance, $1 / \mathrm{m}^{2}$ ) and fluid porosity (1-fabric porosity) were defined for the fabric.

The preform properties of the multilayer interlocked fabrics used for the present study have already been studied for dependency on the structural factor, the $I$. The porosity and permeability values of the multilayer preforms published in the article were used as input data for this multiphase simulation model. No slip boundary conditions were set (default) for the walls of both phases. For the resin phase, 1 was set under the volume fraction for inlet and 0 was set for back-flow volume fraction for outlet boundary conditions.

\subsection{Solution Initiation and Monitoring}

Solution controls were defined for the flow calculation and the relaxation factors for pressure $(0.3)$, density (1), body forces (1), momentum (0.1) and volume fraction (0.3) were defined. Discretization schemes for pressure (standard), pressure-velocity coupling (PISO), momentum (first order upwind) and volume fraction (first order upwind) were selected for the solution control. Solution initialization was set from the outlet, relative to the cell reference frame. A converged solution was defined for the residual of magnitude (0.001) for continuity and velocities ( $\mathrm{x}$, $\mathrm{y}$ and $\mathrm{z}$ ). The residuals and inlet/outlet mass flow rate were monitored during computation. Animation monitoring and frame recordings were set for resin volume fraction images for the fabric (interior), inlet and outlet at defined time steps, so that a video can be made of the flow front through streaming the frames.

\subsection{Calculations}

The computation of the solution was commenced by iterating time steps at 0.01 second for 4000 time steps (for fabric of $1 \mathrm{~mm}$ thickness) and 2000 time steps (for fabric thickness of $1.5 \mathrm{~mm}$ ) with 3 iterations for each time step. The solution converged to the defined tolerances around 
500-550 iterations for all multiphase simulation of RTM resin flow in multilayer fabrics with thicknesses of $1 \mathrm{~mm}$ and $1.5 \mathrm{~mm}$. A representative residual convergence plot of the N2P1 fabric (1.5 $\mathrm{mm}$ in thickness) is shown by Figure 5.

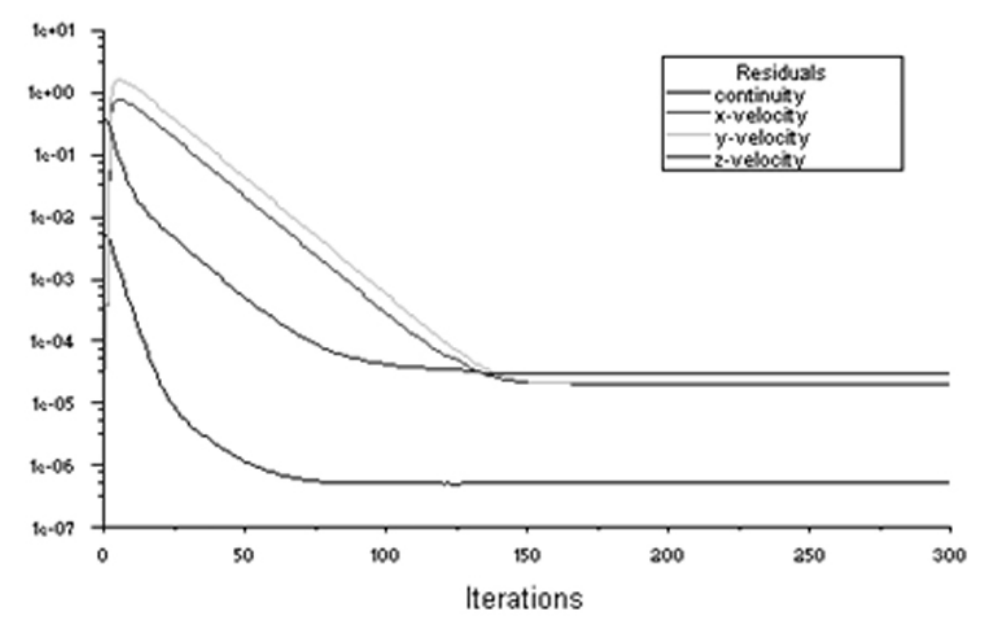

Fig. 5. Residual Plot of N2P1 (1.5mm)

\section{Experimental RTM Set-up}

An ever increasing demand for faster composite manufacture has pressed the composite industry to replace hand lay-up techniques with alternative fabrication processes, such as RTM. RTM is defined as a closed molding process of composite preparation in which catalyzed resin is transferred into an enclosed mold cavity to impregnate a pre-positioned fibrous reinforcement (preform).

A type of RTM, called vacuum-assisted resin transfer molding (VARTM), refers to a variety of related processes, in which VARTM draws resin into a preform through the use of a vacuum rather than positive pressure applied to inject resin in common RTM processes. These RTM represent the fastest growing new molding composite manufacture technology (Hazen, 2005). The experimental RTM setup fabricated for the present study is illustrated as shown in Figure 6.

The steps involved in the RTM process are (Verleye, 2008): design and manufacture of the mold, placement of textile reinforcement into the mold and closing the mold, resin flow through the textile preform, hardening of the resin, and opening the mold and taking out the finished composite part.

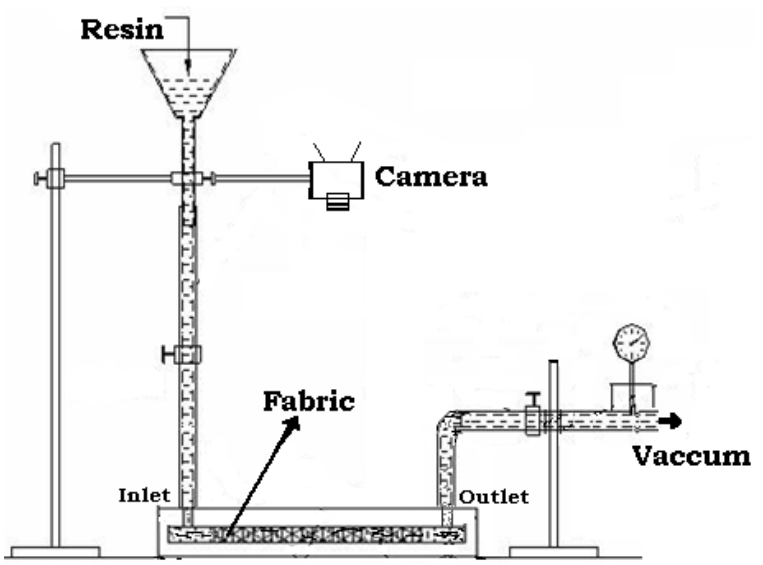

Fig. 6. Experimental RTM setup

Transparent molds made of acrylic polymer were used in the flow experiments. Two molds had flat plates sized $200 \mathrm{~mm}$ by $200 \mathrm{~mm}$ with a thickness of $25 \mathrm{~mm}$, which are shown in Figure 7. Nozzles (inner diameter $4.5 \mathrm{~mm}$ ) were fitted onto the top mold at the inlet and outlet vent locations, $17 \mathrm{~cm}$ away from each other and $1 \mathrm{~cm}$ away from the mold beading groove edge. Thick polyethylene tubes of $3 \mathrm{~mm}$ were used for resin transport from the resin container (Figure 7) to one nozzle end on the mold (resin inlet) and from another nozzle end of the top mold (resin outlet) to the vacuum pump. A vacuum pressure gauge $(0-760 \mathrm{~mm}$ of $\mathrm{Hg}$ ) was used in between the outlet and vacuum pump to 
determine the vacuum pressure value at the outlet. Two aluminum spacers (Figure 7) with thicknesses of $1.5 \mathrm{~mm}$ and $1 \mathrm{~mm}$ were used to maintain constant compaction of the preform between the mold assemblies. A silicon flexible rubber tube (diameter of $1 / 8^{\text {th }}$ of an inch) as beading was used between the spacer and molds to maintain the sealed vacuum conditions in the mold assembly.

All of the RTM flow experiments were conducted with an unsaturated polyester resin system (GP grade) in an uncatalysed form to maintain the transparency of the mold intact. The viscosity of the resin system was found to be $600 \mathrm{cP}$ at $25^{\circ} \mathrm{C}$ (Brookfield viscosity, determined by spindle BS-29 at $25^{\circ} \mathrm{C}, 50 \mathrm{rpm}$ ). A clear view of the flow fronts was recorded during the RTM experiment using a Kodak digital camera (EasyShare C315, 5 megapixels, lens $36 \mathrm{~mm}$ ) mounted on a tripod above the mold assembly. Three trials were taken with each multilayer perform, and the mold fill time and flow front videos were recorded.

\section{Results and Discussions}

Figures 8 and 9 provide the resin mass flow rate plots with respect to flow time obtained by the 3D MM RTM flow simulation for the N2P1 sample with a spacer of $1.5 \mathrm{~mm}$ in thickness. It can be observed from the outlet mass flow rate plot that the resin starts to flow out from the outlet after 8 seconds and after 15 seconds, it reaches a steady rate. An animated frame of the resin volume fraction for the N2P1 $(1.5 \mathrm{~mm})$ fabric at 1 second and 5 seconds are shown in Figure 10.

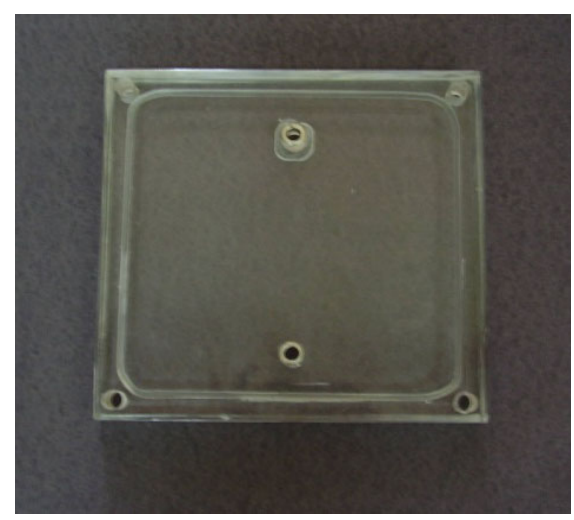

Top Mold

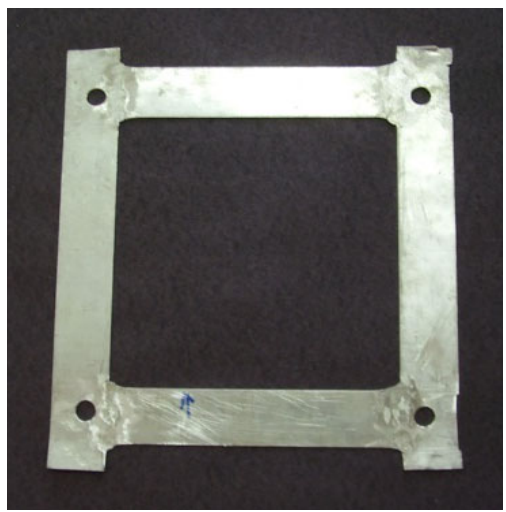

Spacer

Resin container

Fig. 7. Fabricated Parts of RTM Setup 


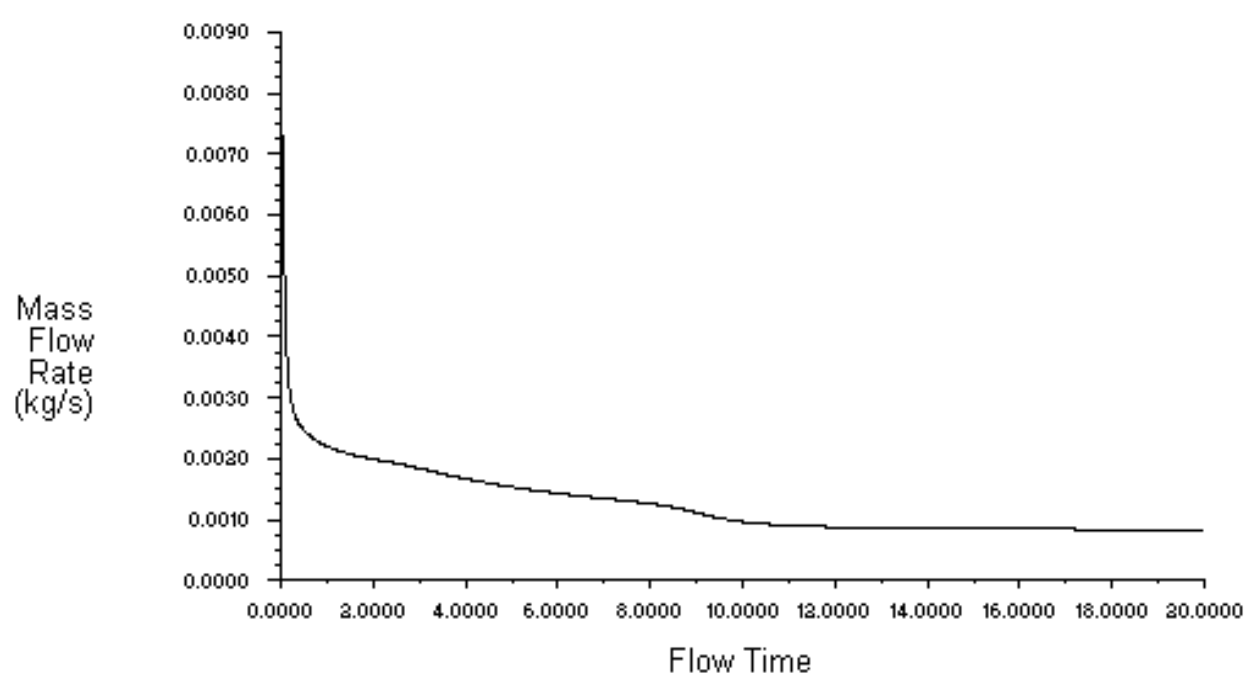

Fig. 8. Mass Flow Rate at Inlet (N2P1-1.5mm)

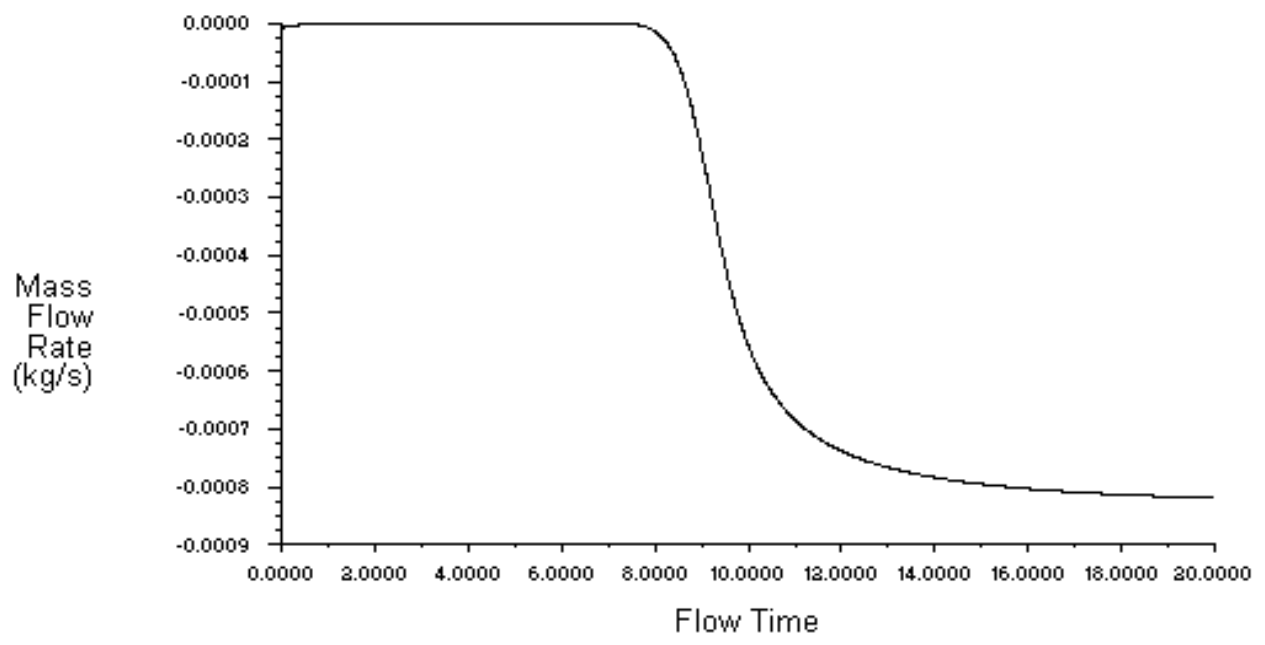

Fig. 9. Mass Flow Rate at Outlet (N2P1-1.5mm)

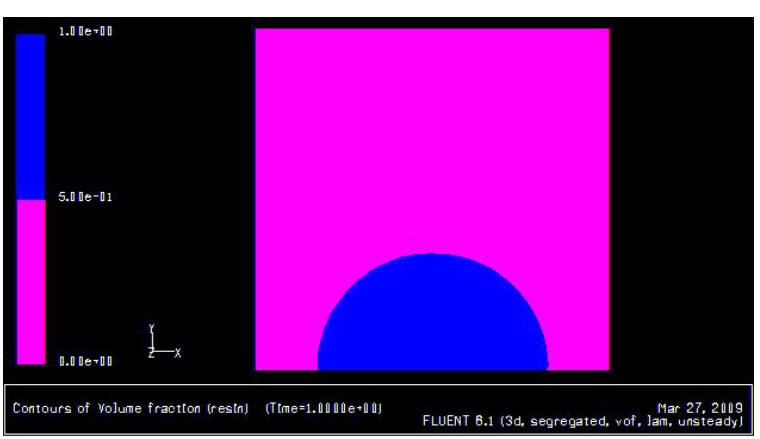

1 Second

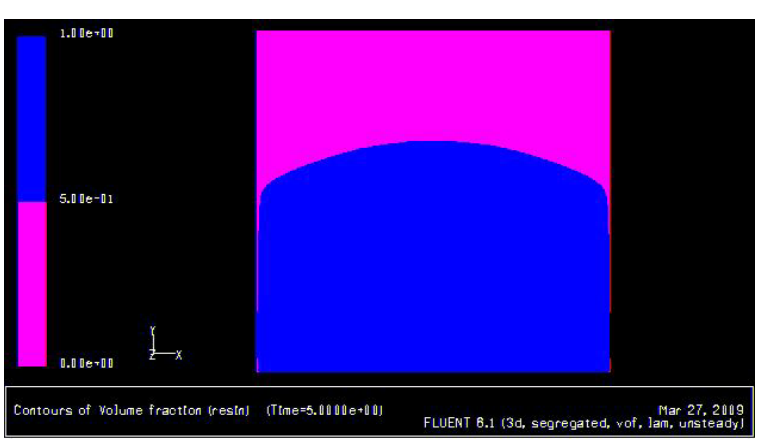

5 Seconds

Fig. 10. Multiphase Model-Contour of Resin Flow in N2P1 (1.5mm) Fabric 
Table 3 (A and B) details the mold filling times recorded for the experimental (Exp) RTM process and $\mathrm{MM}$ for multilayered preforms with thickness spacers of $1 \mathrm{~mm}$ and $1.5 \mathrm{~mm}$. The mold filling times were observed to be shorter for the MM compared to the experimental values.

Table 3 Mold Fill Times of the RTM Experiment and Simulated Models

A- $1 \mathrm{~mm}$ Spacer

\begin{tabular}{cccc}
\hline & Exp & Model & E-\% \\
\hline N2P1 & $28.3(8.2)$ & 24.4 & 16.0 \\
N2P2 & $24.1(3.7)$ & 23.9 & 0.8 \\
N2P3 & $21.6(4.1)$ & 23.7 & 7.2 \\
N2P4 & $19.4(2.2)$ & 18.5 & 4.9 \\
N3P1 & $21.0(5.9)$ & 23.5 & 10.6 \\
N3P2 & $22.3(7.8)$ & 21.1 & 4.7 \\
N3P3 & $21.1(9.1)$ & 24.2 & 13.2 \\
N3P4 & $19.0(4.8)$ & 21.8 & 12.8 \\
\hline
\end{tabular}

B- $1.5 \mathrm{~mm}$ Spacer

\begin{tabular}{cccc}
\hline & Exp & Model & E-\% \\
\hline N2P1 & $12.1(4.3)$ & 16.5 & 26.7 \\
N2P2 & $10.2(3.6)$ & 13.2 & 22.7 \\
N2P3 & $10.9(5.8)$ & 12.1 & 9.9 \\
N2P4 & $10.1(7.1)$ & 10.7 & 5.6 \\
N3P1 & $11.2(8.4)$ & 13.1 & 14.5 \\
N3P2 & $8.9(9.1)$ & 10.6 & 16.0 \\
N3P3 & $11.2(3.9)$ & 11.2 & 0 \\
N3P4 & $10.9(5.4)$ & 11.1 & 1.8 \\
\hline
\end{tabular}

( $\mathrm{CV} \%$ values are given within parenthesis)

The experimental RTM process of N3P5 fabric with $1 \mathrm{~mm}$ and $1.5 \mathrm{~mm}$ spacers showed the highest mold filling time (48 $\mathrm{sec}$ and $31 \mathrm{sec}$, respectively), during which the preform was found to be deforming and jamming the outlet. This event could be due to less interlacement in the N3P5 structure which is responsible for easy structural deformation. Hence, the readings of the N3P5 RTM process have been discarded for result discussions due to experimental error. Errors (E-\%) have been calculated for the mold filling times of the models against the experimental data as presented in the table. The average error of the $\mathrm{MM}$ is $8.8 \%$ for $1 \mathrm{~mm}$ and $12.2 \%$ for $1.5 \mathrm{~mm}$, respectively, which shows that the predictions of the $\mathrm{MM}$ are dependable. The contours of the resin flow front through the N2P1 $(1.5 \mathrm{~mm})$ fabric at 1 second and 5 seconds during the experimental RTM process are shown in Figure 11.

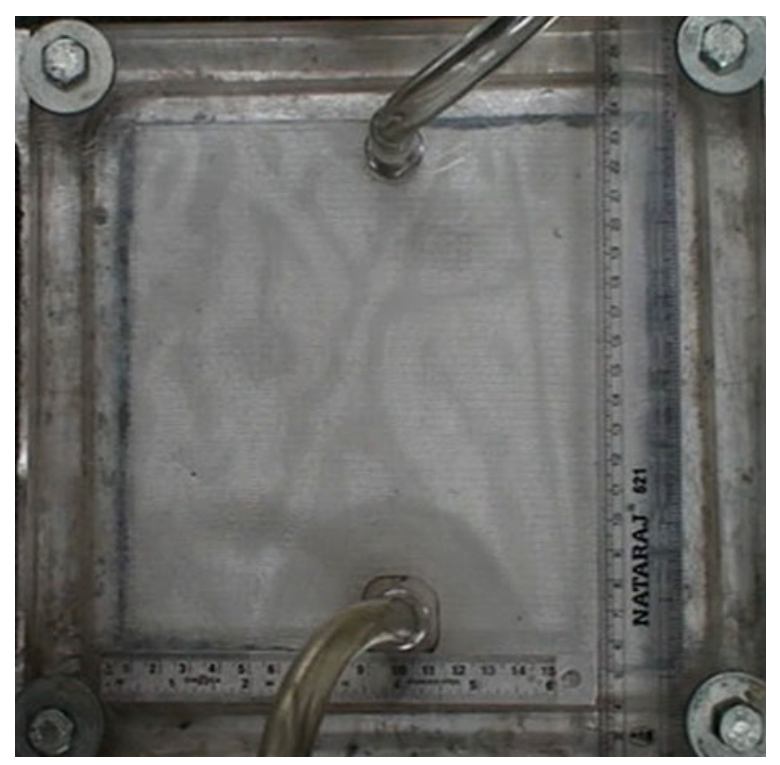

1 Second

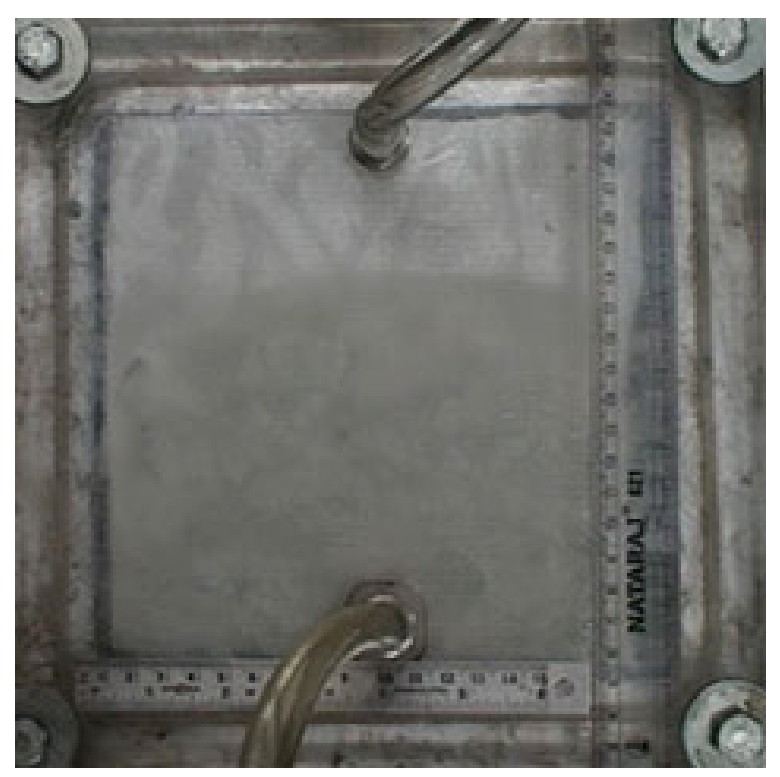

5 Seconds

Fig. 11. Contour of resin flow front in the N2P1 Experiment (1.5mm)

A plot of $I$ against the mold filling time for $1 \mathrm{~mm}$ and $1.5 \mathrm{~mm}$ thicknesses are displayed in Figures 12 and 13, respectively. It can be observed that the mold filling time increases with increased interlacement which can be reasoned as higher interlacements in the preform that induce a higher 
tortuous path of yarn in the structure, thus increasing resistance for the resin flow during the RTM process.

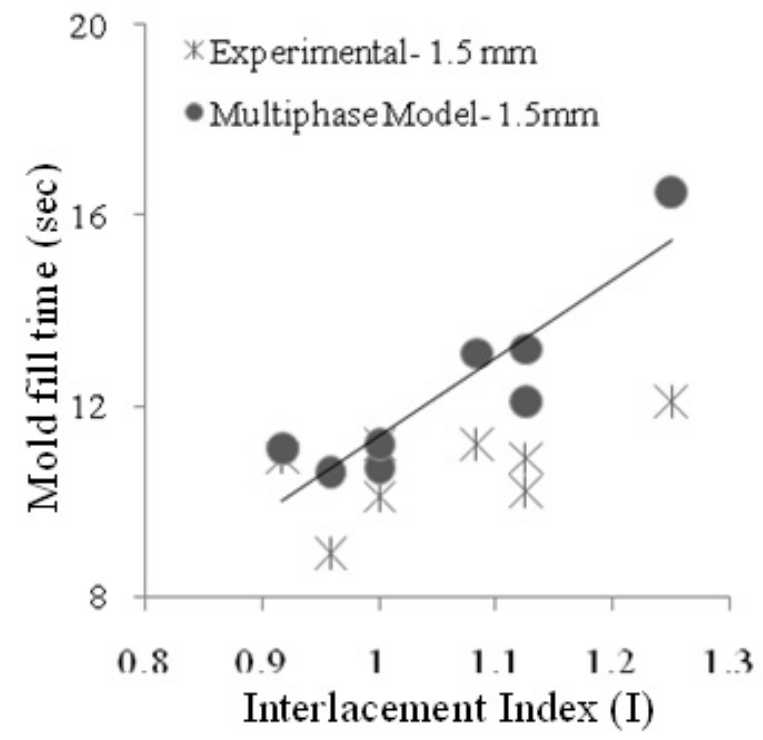

Fig. 12. Influence of Multilayer Structure on Resin Flow Behaviour (1.5mm)

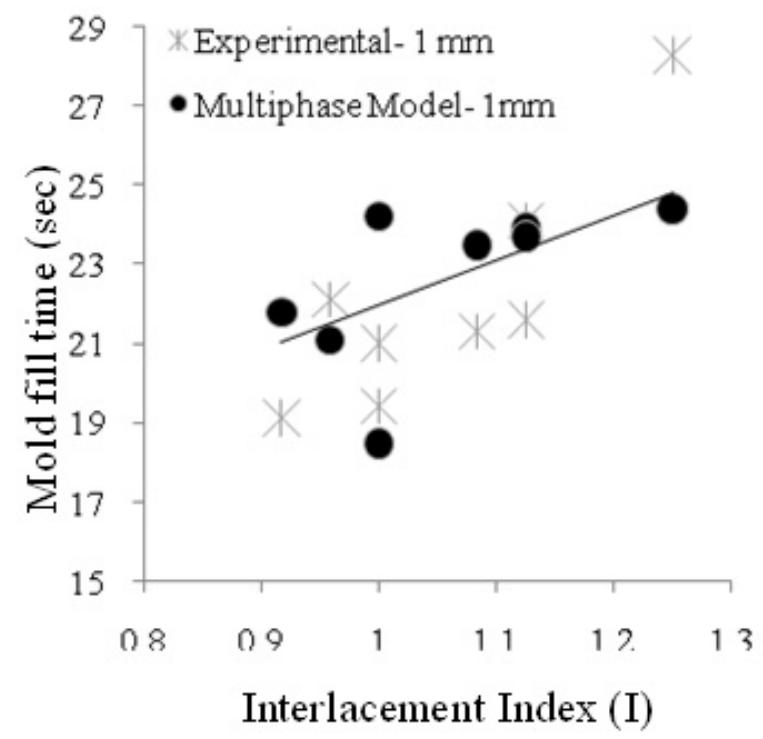

Fig. 13. Influence of Multilayer Structure on Resin Flow Behaviour $(1 \mathrm{~mm})$

\section{Image Analysis of Simulated and Experimental Flow Fronts}

Images of the flow front during the RTM process at 2 seconds were taken from the simulated MM results and experimental videos. The images (Figure 14) were subjected to comparative analyses by an image processing technique which used Image J software. The areas of resin flow in the simulated and experimental frames were calculated and are tabulated in Table 4. It was observed that the flow, at any given time, was generally higher for the MM compared to the experimental results.

Table 4. Resin Flow Area $\left(\mathrm{cm}^{2}\right)$

\begin{tabular}{ccccc}
\hline & \multicolumn{2}{c}{$1.5 \mathrm{~mm}$} & \multicolumn{2}{c}{$1 \mathrm{~mm}$} \\
\cline { 2 - 5 } & Exp & Model & Exp & Model \\
\hline N2P1 & 42.2 & 45.9 & 24.8 & 27.0 \\
N2P2 & 41.4 & 46.9 & 24.4 & 27.6 \\
N2P3 & 41.4 & 42.6 & 24.4 & 25.1 \\
N2P4 & 45.8 & 48.8 & 26.9 & 28.7 \\
N3P1 & 35.9 & 42.8 & 21.1 & 25.2 \\
N3P2 & 44.8 & 50.3 & 26.4 & 29.6 \\
N3P3 & 48.2 & 53.8 & 28.4 & 31.6 \\
N3P4 & 59.7 & 68.7 & 35.1 & 40.4 \\
N3P5 & 42.2 & 45.9 & 24.8 & 27.0 \\
\hline
\end{tabular}

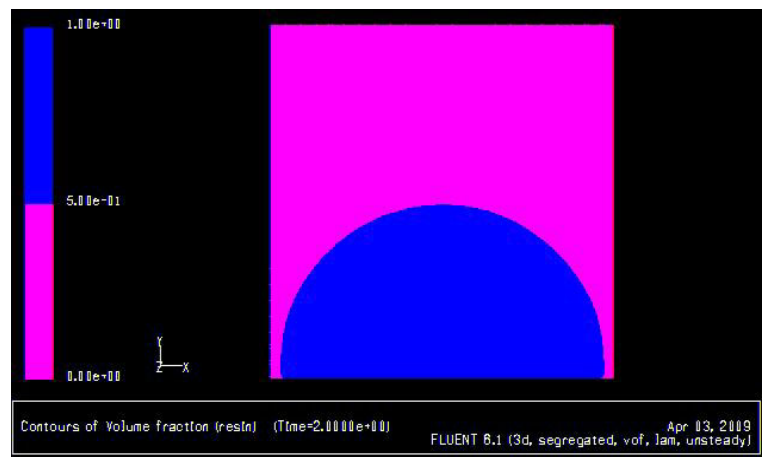

Multiphase simulated flow area

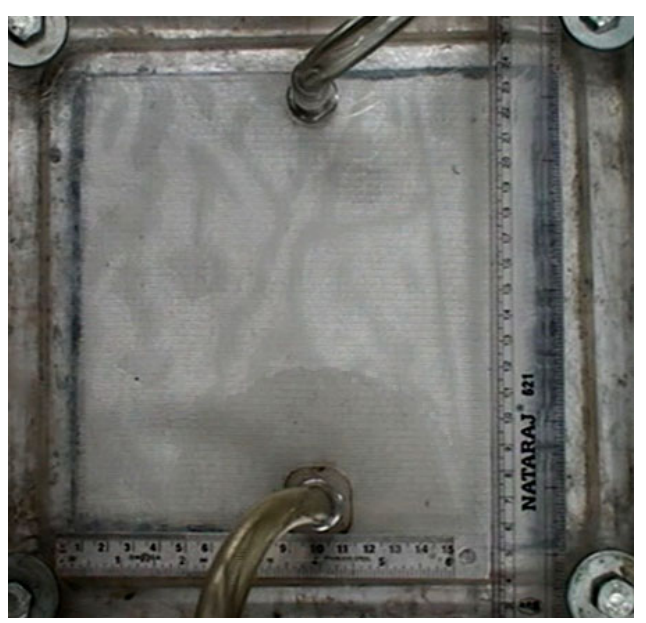

Experimental RTM

Fig. 14. Contour of Resin Flow Front in N2P1 $(1.5 \mathrm{~mm})$ at $2 \mathrm{secs}$ 
A graph of the flow area at a 2 second flow duration against $I$ (Figure 15) shows that the flow area decreases with increased interlacements in the multilayered preforms. This could again, be explained with the level of interlacements in the multilayered structures, as the yarn in fabric will be straighter with lower interlacements, and hence the resin flow will be better for multilayered preforms with a lower $I$.

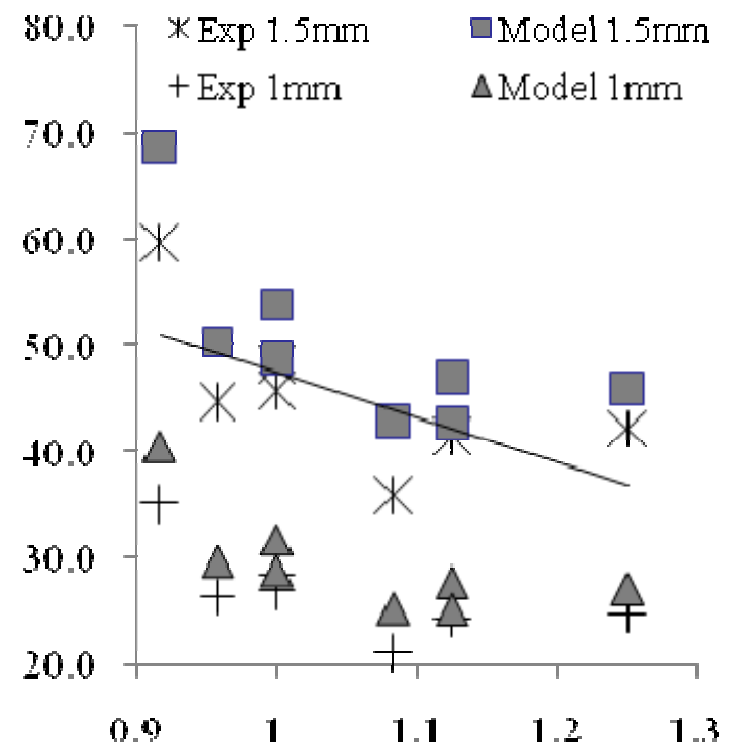

Fig. 15. Influence of Multilayer Structure on the Resin Flow Behaviour

\section{Conclusions}

A 3D multiphase model has been successively developed to predict the time required for RTM mold filling based on the permeability properties of multilayer fabrics. The mold filling time predicted using $\mathrm{MM}$ (error $\% \approx 8.8 \& 12$ ) is found to be reasonable compared to the experimental time for RTM mold filling. A fabric structural factor, $I$, has significant influence on the resin flow behaviour of the preform. A higher $I$ of the perform means longer time taken to fill the mold in both the experimental and simulated results.

\section{REFERENCES}

[1] Adanur, S. \& Tam, A.C. 1997, 'On-machine interlocking of 3D laminate structures for composites', Composites Part B, vol. 28B, pp. $497-506$.

[2] Cairns, D.S., Humbert, D.R. \& Mandell, J.F. 1999, 'Modeling of resin transfer molding of composite materials with oriented unidirectional plies', Composites: Part A, vol. 30, no. 3, pp. 375-383.

[3] Fluent 6.1 tutorial guide. 2003, vol. 1 \& 2, Fluent Inc., Lebanon.

[4] Hu, H. 2000, 'Comparison between laminated and integrated glass fibre reinforced plastics', Materials \& Design, vol. 21, pp. 461 - 464.

[5] Hu, H., Zhilli, Z. 2002, 'Tensile behaviour of 3D woven composites by using different fabric structures', Materials \& Design, vol. 23, pp. $671-674$.

[6] Hazen, J.R. 2005, Source Book 2005, Industry overview: Resin transfer molding process, Ray Publishing Inc., Colorado, USA.

[7] Kruckenberg, T. \& Paton, R. 1998, Resin transfer moulding for aerospace structures, Kluwer academic publishers, MA.

[8] Milasius, V. 2000, 'An integrated structure factor for woven fabrics. Part II: The fabric-firmness factor', The Journal of the Textile Institute, vol. 91, pp. 277 - 283.

[9] Padaki, N.V., Alagirusamy, R., Deopura, B.L. \& Fangueiro, R., 'Studies on preform properties of multilayer interlocked woven structures based on fabric geometrical factors', Journal of Industrial Textiles (accepted for publication).

[10] Padaki, N.V., Sugun, B.S., Alagirusamy, R., Deopura, B.L. \& Fangueiro, R. 2008, 'Low velocity impact behaviour of textile reinforced composites', Indian journal of fibre and textile research, vol. 33, no. 2, pp. 189-202.

[11] Patnaik A., Rengasamy R.S., Kothari V.K. \& Ghosh A. 2006, 'Wetting and wicking in fibrous materials', Textile Progress, vol. 38, no. 1 , The Textile Institute, Manchester.

[12] Verleye, B. 2008, 'Computation of the permeability of multi-scale porous media with application to technical textiles', Katholieke Universiteit Leuven, PhD Thesis, ISBN 978-90-5682-913-1.

[13] Weimer, C. 2003, 'Preform-engineering: applied sewing technologies to incorporate part and process functions into dry textile reinforcements', Composites Science and Technology, vol. 63, pp. 2089-2098. 